\title{
Correlations between abnormalities of
} morphological details and DNA fragmentation in human sperm

\author{
Hiep Tuyet Thi Nguyen ${ }^{1}$, Hong Nhan Thi Dang ${ }^{1}$, Thai Thanh Thi Nguyen ${ }^{1}$, Trung Van Nguyen ${ }^{1}$, Thuan Cong Dang ${ }^{2}$, \\ Quoc Huy Vu Nguyen ${ }^{3}$, Minh Tam Le ${ }^{1,3}$
}

${ }^{1}$ Center for Reproductive Endocrinology and Infertility, Departments of ${ }^{2}$ Histology, Embryology, and Pathology and ${ }^{3}$ Obstetrics and Gynecology, Hue University of Medicine and Pharmacy, Hue University, Hue, Vietnam

Objective: As the associations of sperm DNA fragmentation with morphology have not been examined in detail, this study aimed to investigate the relationship between abnormalities of morphological details and DNA integrity in human sperm.

Methods: In this cross-sectional study, men from infertile couples were enrolled at Hue Center for Reproductive Endocrinology and Infertility, Vietnam. Conventional semen parameters, including morphological details, were analyzed following the World Health Organization 2010 criteria. Sperm DNA fragmentation was evaluated using a sperm chromatin dispersion assay. The relationships and correlations between semen parameters, sperm morphology, and the type of halosperm and the DNA fragmentation index (DFI) were analyzed.

Results: Among 130 men in infertile couples, statistically significant differences were not found in the sperm halo type between the normal and abnormal sperm morphology groups. The percentage of round-head spermatozoa was higher in the DFI $>15 \%$ group (16.98\% $\pm 12.50 \%)$ than in the $\mathrm{DFI} \leq 15 \%$ group $(13.13 \% \pm 8.82 \%)$, higher values for amorphous heads were found in the $\mathrm{DFI}>15 \%$ group, and lower values for tapered heads were observed in the DFI $\leq 15 \%$ group; however, these differences were not statistically significant. Small-halo sperm and the DFI were positively correlated with round-head sperm ( $r=0.243, p=0.005$ and $r=0.197, p=0.025$, respectively).

Conclusion: The rate of general sperm morphological abnormalities in semen analysis was not related to sperm DNA integrity. However, round sperm heads were closely associated with sperm DNA fragmentation.

Keywords: Anatomy and histology; DNA fragmentation; Infertility; Male; Spermatozoa

\section{Introduction}

Infertility is a reproductive health problem that affects approximately $15 \%$ of reproductive-aged couples worldwide; male factors

Received: June 21, $2021 \cdot$ Revised: August 13, 2021 · Accepted: September 6, 2021 Corresponding author: Minh Tam Le

Department of Obstetrics and Gynecology, Hue University of Medicine and Pharmacy, Hue University, 6 Ngo Quyen st, Hue, Viet Nam

Tel: +84-23-4626-9696 Fax: +84-23-4382-2173

E-mail: leminhtam@hueuni.edu.vn

*Hiep Tuyet Thi Nguyen was funded by Vingroup Joint Stock Company and was supported by the Domestic Master/ PhD Scholarship Program of Vingroup Innovation Foundation (VINIF), Vingroup Big Data Institute (VINBIGDATA), code VINIF. 2020.TS.44.

This is an Open Access article distributed under the terms of the Creative Commons Attribution Non-Commercial License (http://creativecommons.org/licenses/by-nc/4.0/) which permits unrestricted non-commercial use, distribution, and reproduction in any medium, provided the original work is properly cited. contribute as a unique cause in approximately $30 \%$ of infertility cases [1]. Male factor infertility results from pre-testicular, testicular, or post-testicular abnormalities. However, in some cases, no reason for diminished sperm quality can be identified [2]. Conventional semen analysis is routinely performed according to standard criteria in the World Health Organization (WHO) guidelines and is accepted as the first step in evaluating male fertility [3]. Further, sperm DNA fragmentation (SDF) tests have recently gained interest as a way to assess sperm quality in the context of recurrent miscarriage, unexplained infertility, varicocele, or recurrent implantation failure after in vitro fertilization/intracytoplasmic sperm injection (IVF/ICSI) [2].

During spermiogenesis, when spermatids transform into spermatozoa, the chromatin of sperm cells can be damaged by endogenous and exogenous factors $[4,5]$. Due to chromatin compaction, the substantial reduction in cell cytoplasm leads to a limited self-repair pro- 
cess for fragmentation [5]. Severe DNA fragmentation may occur after spermatogenesis is completed, during the time in the epididymis and other ducts. Lifestyle-related risk factors (e.g., alcohol consumption and smoking, or toxic and hot environments) may also lead to increased concentrations of oxygen free radicals, thereby increasing the levels of sperm DNA damage [6-8]. SDF is correlated with apoptosis and dysfunction of sperm mitochondrial membrane potential, which is in turn negatively related to motility and normal morphology [9].

A high percentage of abnormal sperm, especially abnormalities of structural details, may result from a defective mechanism associated with spermatogenesis and/or sperm maturation. Abnormal sperm morphology is associated with a decrease in routine semen parameters [10] as well as signs of sperm damage (e.g., DNA fragmentation levels) and increased concentrations of reactive oxygen species (ROS) [11].

To date, the relationship between sperm morphology and SDF remains unclear. Furthermore, very few results have been obtained from fresh or prepared samples using different techniques, and sperm morphology has not been assessed in detail [12-14]. Additional information on the detailed characteristics of sperm morphology and information regarding sperm cells may be helpful for predicting sperm quality and advising patients. Therefore, the goal of this study was to determine the relationship between abnormalities of morphological details and DNA integrity of human spermatozoa using the sperm chromatin dispersion (SCD) assay.

\section{Methods}

\section{Study design}

A cross-sectional study was conducted at the Center for Reproductive Endocrinology and Infertility, Hue University of Medicine and Pharmacy, Vietnam, from April 2020 to October 2020. The inclusion criteria were men from infertile couples, with semen analysis and SCD test results. The exclusion criteria were patients with retrograde ejaculation, severe oligospermia (under 1 million/mL), azoospermia, sperm from cryopreservation, or sperm retrieved by surgery. Men with general urogenital infections or a history of inguinal hernia were also excluded. This study was approved by the Ethics Committee of Hue University of Medicine and Pharmacy (No. 678a/QĐĐHYD). All patients agreed to participate in this study and signed an informed consent form.

\section{Semen analysis}

Fresh semen samples were obtained and tested in accordance with the 2010 WHO standards [3]. Sperm motility was analyzed manually using phase-contrast microscopy at $\times 400$ magnification; sperm viability was assessed by eosin staining. For morphological as- sessment, after staining with Giemsa, the morphology of the sperm head shape, acrosomal region, sperm neck, midpiece, tail, and cytoplasmic droplets was determined under a microscope at $\times 1,000$ magnification, according to the WHO 2010 guidelines. Head defects included abnormal head shapes and vacuolation, abnormal acrosomal areas, double heads, or any combination thereof. Neck and midpiece defects included a folding neck, an irregular thick, thin or a sharply bent midpiece. Principal piece defects included short, multiple, broken, smooth hairpin bends, sharply angulated bends, an irregular width, coiled structure, or any combination thereof. Excess residual cytoplasm (ERC) was recorded when the cytoplasm was larger than one-third of the sperm head size [3]. The images of abnormal sperm were taken using an Olympus CX41 microscope (Cebu, Philippines) and Infinity 1 Lumenera software (Ottawa, Canada) and shown in Figure 1. Based on the morphology results, the samples were separated into normal (normal morphology rate $\geq 4 \%$ ) and abnormal (normal morphology rate < 4\%) morphology groups.

\section{SCD test}

All fresh semen samples were tested for fragmented DNA using the Halosperm HT-HS10 system (Halotech DNA, Madrid, Spain). A 20$\mu \mathrm{L}$ sperm sample was added to $40 \mu \mathrm{L}$ of low-melting agarose and mixed evenly. Subsequently, an $8-\mu \mathrm{L}$ aliquot was placed on a slide and refrigerated at $4^{\circ} \mathrm{C}$ for 10 minutes to solidify the agarose. The slide was then immersed respectively in an acid denaturant for 7 minutes and lysis solution for 25 minutes. After that, the slide was rinsed in distilled water for 5 minutes, dehydrated in ethanol baths at concentrations of $70 \%$ ( 2 minutes) and 100\% ( 2 minutes), and airdried. Giemsa staining was used to stain the sperm at the end. The slides were examined under phase-contrast microscopy at $\times 1,000$ magnification. The DNA fragmentation index (DFI) was estimated as the sum of DNA fragmentation spermatozoa per 500 spermatozoa. Spermatozoa without DNA fragmentation included sperm cells with a large halo (halo width equal to or higher than the diameter of the core) or medium halo (halo size between large and small). Spermatozoa with fragmented DNA included any sperm cells with small halos (halo width equal to or smaller than one-third of the diameter of the core) or no halo, as well as those that were degraded (no halo and presenting a core that was irregularly or weakly stained). To prevent bias, the slide observations were recorded and then assessed by two experienced andrologists (NTHT and DTHN). The images of sperm halos were taken using an Olympus CX41 microscope and Infinity 1 Lumenera software, as shown in Figure 2. The normal DFI group was defined as those with a DFI $\leq 15 \%$, while a DFI $>15 \%$ was considered abnormal. 

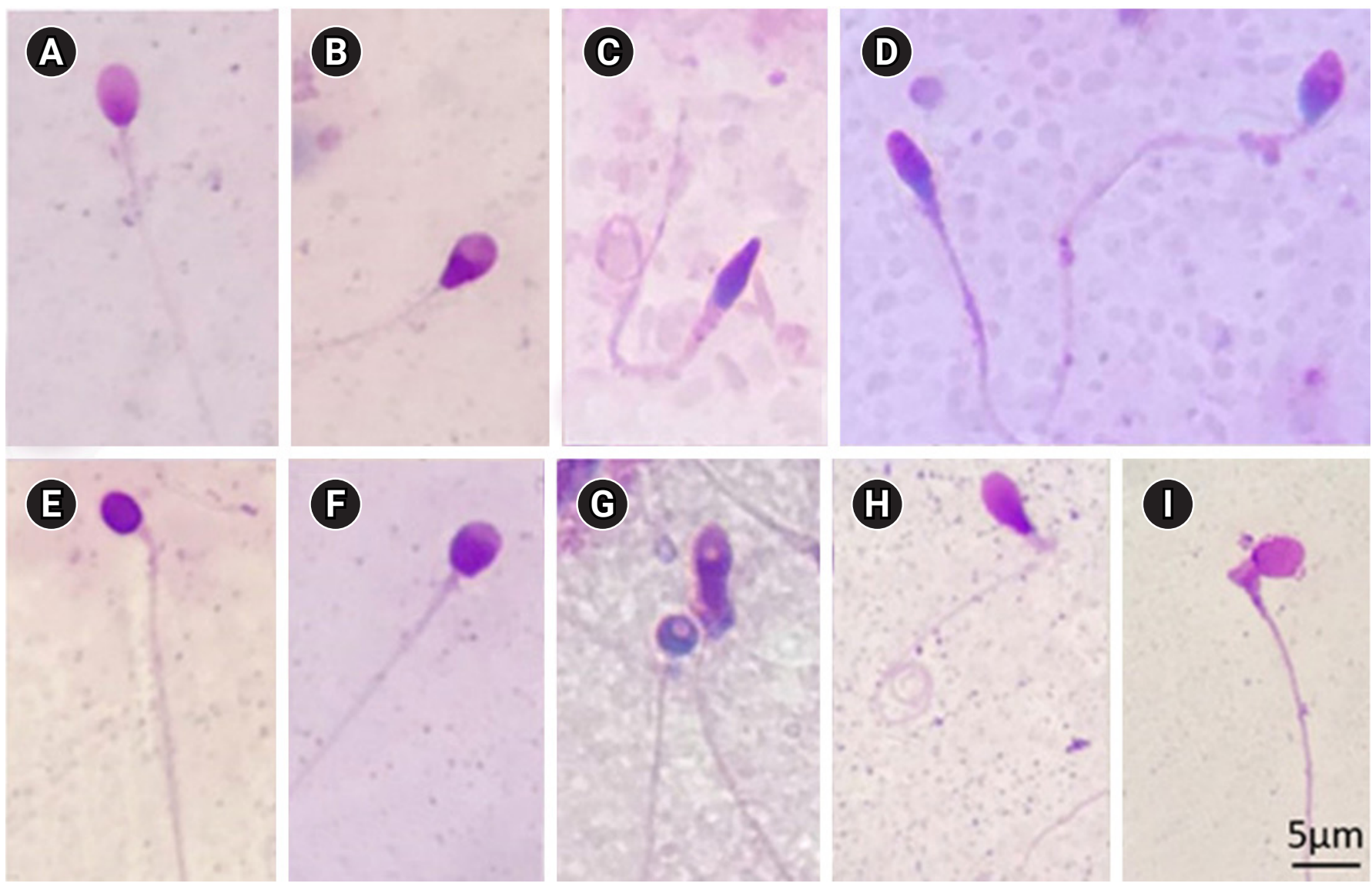

Figure 1. Images of abnormal sperm. (A) Normal sperm cell. (B) Pyriform-head sperm cell. (C) Sperm cell with a tapered head, thick insertion midpiece, and coiled tail. (D) Tapered-head sperm cell and an amorphous-head sperm cell. (E) Round-head sperm cell without an acrosome. (F) Sperm cell with a small acrosomal area. (G) Round-head sperm cell with vacuolation and an amorphous-head sperm cell with vacuolation and excess residual cytoplasm. (H) Pyriform sperm head with bent midpiece and coiled tail. (I) Sperm cell with a bent neck and excess residual cytoplasm greater than one-third of the head. (Giemsa stain, x1,000)
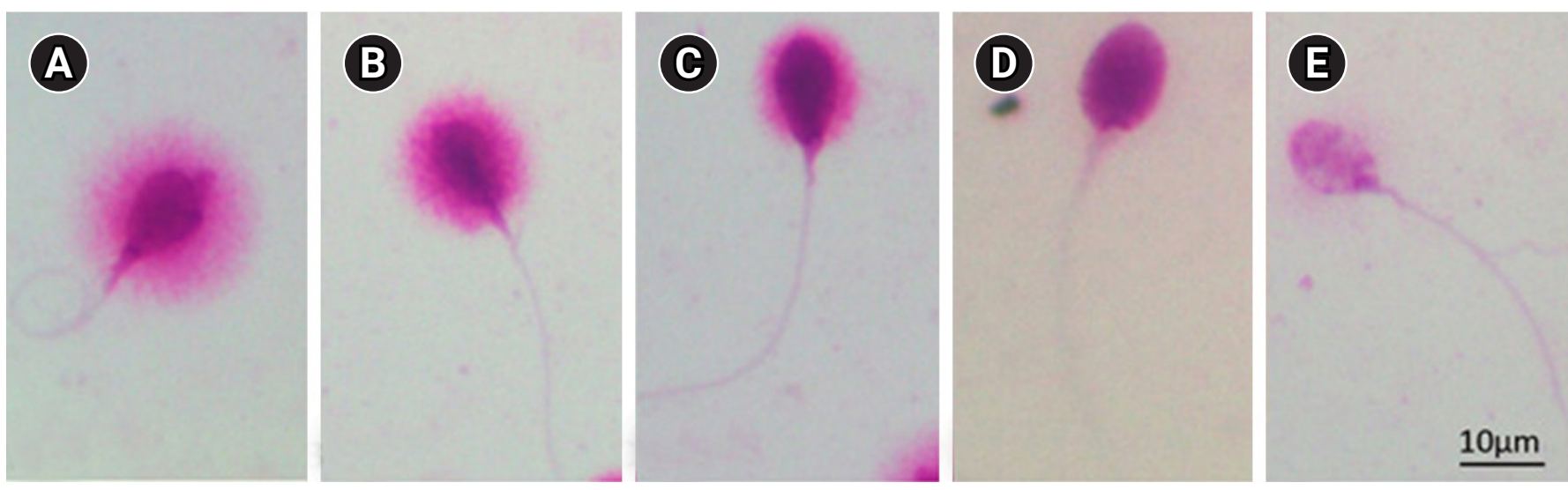

Figure 2. Classification of human sperm DNA fragmentation using the Halosperm test. The normal group included (A) sperm with a large halo and (B) sperm with a medium halo; while the abnormal group included (C) sperm with a small halo, (D) sperm without a halo, and (E) degraded sperm. (Giemsa stain, $x 1,000)$ 


\section{Statistical analysis}

All numerical data were presented as mean \pm standard deviation. Frequencies were expressed as percentages, and the mean values were compared between two groups using analysis of variance. The Pearson correlation coefficient $(r)$ was used to determine the association between values of sperm cells and the DFI. Differences between values were considered statistically significant at $p<0.05$. IBM SPSS ver. 20.0 (IBM Corp., Armonk, NY, USA) was used for statistical analyses.

\section{Results}

In total, 130 semen samples from 130 infertile men were collected for this study. As shown in Table 1, they ranged in age from 25 to 51 years old (mean, $34.16 \pm 5.72$ years). The following results were obtained for semen parameters: semen volume, $2.33 \pm 0.88 \mathrm{~mL} ; \mathrm{pH}$, $7.31 \pm 0.47$; sperm progressive motility $(\mathrm{PR}), 28.55 \% \pm 9.88 \%$; concentration, $34.35 \pm 18.34 \times 10^{6} / \mathrm{mL}$; vitality, $83.02 \% \pm 9.06 \%$; normal morphology, $4.75 \% \pm 3.06 \%$; and sperm DFI, $34.57 \% \pm 22.42 \%$.
Statistically significant differences were found between the normal and aberrant morphology groups in terms of PR $(31.35 \% \pm 9.13 \%$ vs. $24.73 \% \pm 9.66 \%, p=0.000)$, sperm concentration $(38.40 \pm 18.08$ $\times 10^{6} / \mathrm{mL}$ vs. $\left.28.84 \pm 17.37 \times 10^{6} / \mathrm{mL}, p=0.003\right)$, viability rate $(84.81 \% \pm 5.31 \%$ vs. $80.58 \% \pm 12.11 \%, p=0.018)$, abnormal head rate $(85.20 \% \pm 7.73 \%$ vs. $91.96 \% \pm 7.27 \%, p=0.00)$, pyriform rate $(18.41 \% \pm 10.44 \%$ vs. $12.56 \% \pm 10.74 \%, p=0.002)$, and ERC rate $(0.73 \% \pm 1.43 \%$ vs. $3.20 \% \pm 4.69 \%, p=0.000)$. Amorphous morphology was the most common head abnormality, at $29.66 \% \pm 13.46 \%$; however, only a trend towards significance was observed between the two morphological groups $(p=0.051)$. Regarding the characteristics of SDF, no statistically significant differences in the halo characteristics were found between the normal and abnormal morphology groups.

Table 2 shows the results for the relationship of semen characteristics with the two groups (DFI $\leq 15 \%$ and $\mathrm{DFI}>15 \%$ ); the results revealed no statistically significant difference between the two groups in terms of routine semen parameters and abnormalities of morpho-

Table 1. Comparison of semen parameters and sperm DFI between normal and abnormal sperm morphology groups

\begin{tabular}{|c|c|c|c|c|}
\hline Characteristics & $\begin{array}{c}\text { Total } \\
(n=130)\end{array}$ & $\begin{array}{l}\text { Normal morphology } \\
(n=75)\end{array}$ & $\begin{array}{l}\text { Abnormal morphology } \\
\qquad(\mathrm{n}=55)\end{array}$ & $p$-value \\
\hline \multicolumn{5}{|l|}{ Semen parameter } \\
\hline Volume (mL) & $2.33 \pm 0.88(1-5)$ & $2.33 \pm 0.82$ & $2.35 \pm 0.97$ & 0.907 \\
\hline $\mathrm{pH}$ & $7.31 \pm 0.47(6-9)$ & $7.27 \pm 0.45$ & $7.36 \pm 0.53$ & 0.286 \\
\hline PR (\%) & $28.55 \pm 9.88(0-56)$ & $31.35 \pm 9.13$ & $24.73 \pm 9.66$ & 0.000 \\
\hline Concentration $\left(10^{6} / \mathrm{mL}\right)$ & $34.35 \pm 18.34(2-80)$ & $38.40 \pm 18.08$ & $28.84 \pm 17.37$ & 0.003 \\
\hline Viability (\%) & $83.02 \pm 9.06(8-93)$ & $84.81 \pm 5.31$ & $80.58 \pm 12.11$ & 0.018 \\
\hline \multicolumn{5}{|l|}{ Sperm morphology } \\
\hline Normal morphology (\%) & $4.75 \pm 3.06(0-14)$ & $6.77 \pm 2.43$ & $2.00 \pm 0.94$ & 0.000 \\
\hline Abnormal head (\%) & $88.06 \pm 8.23(58-99)$ & $85.20 \pm 7.73$ & $91.96 \pm 7.27$ & 0.000 \\
\hline Amorphous (\%) & $29.66 \pm 13.46(4-72)$ & $27.69 \pm 11.45$ & $32.35 \pm 15.50$ & 0.051 \\
\hline Tapered (\%) & $18.59 \pm 15.72(0-71)$ & $13.21 \pm 12.15$ & $25.93 \pm 17.11$ & 0.000 \\
\hline Round head (\%) & $16.03 \pm 11.79(1-60)$ & $17.17 \pm 11.72$ & $14.47 \pm 11.82$ & 0.198 \\
\hline Pyriform (\%) & $15.94 \pm 10.92(0-49)$ & $18.41 \pm 10.44$ & $12.56 \pm 10.74$ & 0.002 \\
\hline Small acrosomal area (\%) & $7.32 \pm 8.23(0-34)$ & $6.64 \pm 6.94$ & $8.24 \pm 9.703$ & 0.301 \\
\hline Vacuolated (\%) & $2.84 \pm 5.11(0-34)$ & $3.47 \pm 6.03$ & $1.98 \pm 3.35$ & 0.077 \\
\hline Neck and midpiece defects (\%) & $40.96 \pm 15.00(12-81)$ & $39.43 \pm 13.08$ & $43.05 \pm 17.19$ & 0.193 \\
\hline Tail defects (\%) & $22.24 \pm 11.28(2-80)$ & $21.68 \pm 9.94$ & $23.00 \pm 12.94$ & 0.529 \\
\hline ERC (\%) & $2.15 \pm 3.87(0-23)$ & $0.73 \pm 1.43$ & $3.20 \pm 4.69$ & 0.000 \\
\hline \multicolumn{5}{|l|}{ Sperm DNA fragmentation } \\
\hline DFI (\%) & $34.57 \pm 22.42(6-90.6)$ & $34.16 \pm 23.34$ & $35.12 \pm 21.32$ & 0.812 \\
\hline Big halo (\%) & $27.82 \pm 28.45(0-90.20)$ & $27.71 \pm 28.68$ & $27.98 \pm 28.40$ & 0.958 \\
\hline Medium halo (\%) & $37.60 \pm 19.65(2.8-79.6)$ & $38.12 \pm 19.69$ & $36.89 \pm 19.75$ & 0.728 \\
\hline Small halo (\%) & $17.85 \pm 12.98(2.4-54.6)$ & $17.26 \pm 12.39$ & $18.65 \pm 13.83$ & 0.548 \\
\hline Without halo (\%) & $11.86 \pm 11.80(0.8-58.6)$ & $12.41 \pm 13.06$ & $11.12 \pm 9.90$ & 0.542 \\
\hline Degraded (\%) & $4.85 \pm 4.04(0-20.4)$ & $4.49 \pm 3.78$ & $5.33 \pm 4.35$ & 0.241 \\
\hline
\end{tabular}

Values are presented as mean \pm standard deviation (range) or mean \pm standard deviation. DFI, DNA fragmentation index; PR, progressive motility; ERC, excess residual cytoplasm. 
Table 2. Comparison semen parameters and detailed sperm morphology in two groups of sperm DFI

\begin{tabular}{lccc}
\hline Characteristics & DFI $\leq 15 \%(\mathrm{n}=32)$ & DFI $>15 \%(\mathrm{n}=98)$ & $p$-value \\
\hline Volume $(\mathrm{mL})$ & $2.45 \pm 0.82$ & $2.30 \pm 0.903$ & 0.385 \\
$\mathrm{pH}$ & $7.42 \pm 0.42$ & $7.27 \pm 0.484$ & 0.111 \\
PR (\%) & $29.16 \pm 7.58$ & $28.35 \pm 10.55$ & 0.638 \\
Concentration $\left(10^{6} / \mathrm{mL}\right)$ & $32.16 \pm 15.89$ & $35.07 \pm 19.09$ & 0.437 \\
Viability (\%) & $84.66 \pm 7.00$ & $82.49 \pm 9.60$ & 0.242 \\
Normal morphology (\%) & $5.09 \pm 2.57$ & $4.64 \pm 3.20$ & 0.471 \\
Abnormal head (\%) & $87.69 \pm 9.86$ & $88.18 \pm 1.67$ & 0.768 \\
Amorphous (\%) & $27.13 \pm 11.48$ & $30.49 \pm 4.00$ & 0.221 \\
Tapered (\%) & $22.53 \pm 20.19$ & $17.31 \pm 13.83$ & 0.180 \\
Round-head spermatozoa (\%) & $13.13 \pm 8.82$ & $16.98 \pm 12.50$ & 0.059 \\
Pyriform (\%) & $16.53 \pm 11.48$ & $15.74 \pm 10.78$ & 0.725 \\
Small acrosomal area (\%) & $6.56 \pm 6.34$ & $7.56 \pm 8.77$ & 0.553 \\
Vacuolated (\%) & $2.50 \pm 4.95$ & $2.95 \pm 5.19$ & 0.668 \\
Neck and midpiece defects (\%) & $41.30 \pm 15.61$ & 0.658 \\
Tail defects (\%) & $39.94 \pm 13.11$ & $22.10 \pm 12.08$ & 0.810 \\
ERC (\%) & $22.66 \pm 8.50$ & $2.20 \pm 4.046$ & 0.797 \\
\hline
\end{tabular}

Values are presented as mean \pm standard deviation.

DFI, DNA fragmentation index; PR, progressive motility; ERC, excess residual cytoplasm.

Table 3. The correlation between the classification of halo and semen parameters

\begin{tabular}{|c|c|c|c|c|c|c|}
\hline Characteristics & Big halo & Medium halo & Small halo & Without halo & Degraded & DFI \\
\hline \multicolumn{7}{|l|}{ Volume } \\
\hline$r$ & 0.081 & -0.033 & -0.122 & 0.044 & -0.148 & -0.074 \\
\hline$p$-value & 0.360 & 0.713 & 0.167 & 0.620 & 0.092 & 0.402 \\
\hline \multicolumn{7}{|l|}{$\mathrm{pH}$} \\
\hline$r$ & $0.189^{\mathrm{a})}$ & -0.144 & -0.045 & -0.150 & -0.053 & -0.114 \\
\hline$p$-value & 0.031 & 0.103 & 0.611 & 0.089 & 0.546 & 0.195 \\
\hline \multicolumn{7}{|l|}{ PR } \\
\hline$r$ & -0.027 & 0.170 & 0.000 & -0.104 & $-0.332^{b)}$ & -0.115 \\
\hline$p$-value & 0.761 & 0.053 & 0.999 & 0.237 & 0.000 & 0.194 \\
\hline \multicolumn{7}{|l|}{ Concentration } \\
\hline$r$ & 0.041 & 0.024 & -0.077 & -0.018 & -0.099 & -0.072 \\
\hline$p$-value & 0.647 & 0.789 & 0.383 & 0.836 & 0.261 & 0.414 \\
\hline \multicolumn{7}{|l|}{ Vitality } \\
\hline$r$ & 0.044 & 0.150 & 0.014 & $-0.301^{b)}$ & $-0.205^{\mathrm{a})}$ & $-0.188^{a)}$ \\
\hline$p$-value & 0.618 & 0.088 & 0.875 & 0.000 & 0.019 & 0.033 \\
\hline
\end{tabular}

DFI, DNA fragmentation index; PR, progressive motility.

${ }^{\text {a) }}$ The correlation is significant at the 0.05 level; ${ }^{\text {b) }}$ The correlation is significant at the 0.01 level.

logical details. Table 3 presents the relationship between DNA fragmentation characteristics and semen parameters. A weak positive correlation was found between large halos and semen $\mathrm{pH}$. Further, the absence of a halo was negatively correlated with viability ( $r=$ $-0.301, p=0.000$ ), and the incidence of degraded sperm was also negatively correlated with the rate of PR $(r=-0.332, p=0.000)$ and viability $(r=-0.205, p=0.019)$; in general, the DFI was negatively correlated with the viability of spermatozoa $(r=-0.188, p=0.033)$.

The correlations between the classification of halo types and $a b$ normal morphology are shown in Table 4. Round-head spermatozoa had a positive correlation with small-halo sperm and the DFI ( $r=0.243, p=0.005$ and $r=0.197, p=0.025)$, respectively. There were no correlations between other halo characteristics and abnormal sperm morphology. 
Table 4. The correlation between the halo types and detailed sperm morphology abnormalities

\begin{tabular}{|c|c|c|c|c|c|c|c|}
\hline Characteristics & & Big halo & Medium halo & Small halo & Without halo & Degraded & DFI \\
\hline \multirow[t]{2}{*}{ Normal morphology } & $r$ & 0.039 & -0.033 & 0.030 & -0.026 & -0.136 & -0.021 \\
\hline & $p$-value & 0.660 & 0.712 & 0.734 & 0.768 & 0.124 & 0.814 \\
\hline \multirow[t]{2}{*}{ Abnormal head } & $r$ & -0.104 & 0.116 & 0.003 & 0.004 & 0.150 & 0.031 \\
\hline & $p$-value & 0.237 & 0.188 & 0.977 & 0.963 & 0.089 & 0.729 \\
\hline \multirow[t]{2}{*}{ Tapered } & $r$ & 0.106 & -0.039 & -0.159 & -0.040 & 0.070 & -0.100 \\
\hline & $p$-value & 0.231 & 0.659 & 0.071 & 0.655 & 0.428 & 0.258 \\
\hline \multirow[t]{2}{*}{ Pyriform } & $r$ & -0.055 & 0.141 & -0.017 & -0.053 & -0.093 & -0.054 \\
\hline & $p$-value & 0.537 & 0.109 & 0.850 & 0.547 & 0.293 & 0.538 \\
\hline \multirow[t]{2}{*}{ Round-head spermatozoa } & $r$ & -0.125 & -0.044 & $0.243^{\mathrm{a})}$ & 0.091 & 0.046 & $0.197^{b)}$ \\
\hline & $p$-value & 0.157 & 0.618 & 0.005 & 0.303 & 0.600 & 0.025 \\
\hline \multirow[t]{2}{*}{ Amorphous } & $r$ & -0.108 & 0.140 & 0.002 & 0.025 & 0.000 & 0.014 \\
\hline & $p$-value & 0.223 & 0.112 & 0.985 & 0.781 & 0.997 & 0.875 \\
\hline \multirow[t]{2}{*}{ Vacuolated } & $r$ & -0.009 & -0.028 & -0.030 & 0.065 & 0.107 & 0.036 \\
\hline & $p$-value & 0.922 & 0.749 & 0.733 & 0.464 & 0.228 & 0.686 \\
\hline \multirow[t]{2}{*}{ Small acrosomal area } & $r$ & -0.013 & -0.005 & 0.063 & -0.053 & 0.066 & 0.020 \\
\hline & $p$-value & 0.887 & 0.954 & 0.479 & 0.550 & 0.453 & 0.817 \\
\hline \multirow[t]{2}{*}{ Neck and midpiece defects } & $r$ & -0.010 & 0.115 & -0.139 & 0.032 & -0.140 & -0.089 \\
\hline & $p$-value & 0.914 & 0.191 & 0.115 & 0.718 & 0.111 & 0.314 \\
\hline \multirow[t]{2}{*}{ Tail defect } & $r$ & 0.054 & -0.036 & -0.107 & 0.083 & -0.100 & -0.036 \\
\hline & $p$-value & 0.545 & 0.681 & 0.227 & 0.346 & 0.257 & 0.684 \\
\hline \multirow[t]{2}{*}{ ERC } & $r$ & -0.001 & -0.084 & 0.083 & 0.020 & 0.087 & 0.075 \\
\hline & $p$-value & 0.995 & 0.339 & 0.345 & 0.819 & 0.323 & 0.398 \\
\hline
\end{tabular}

DFI, DNA fragmentation index; ERC, excess residual cytoplasm.

${ }^{\text {a) }}$ The correlation is significant at the 0.01 level; ${ }^{\text {b) }}$ The correlation is significant at the 0.05 level.

\section{Discussion}

Sperm morphology is the most relevant parameter in conventional semen analysis for predicting fertilization potential [11,15], a prerequisite factor for the successful use of assisted reproductive technology (ART) $[16,17]$. Our results indicated that abnormal sperm mainly showed changes in head morphology, and that amorphous-head and tapered spermatozoa were more common in the abnormal morphology group. Amorphous heads, tapered heads, and microheads were also reported to predominate abnormal sperm morphology in previous studies $[18,19]$. However, the relationship between the level of SDF and semen parameters has remained unclear. In some studies, the DFI was associated with some semen parameters such as sperm viability [20], motility [21,22], concentration, and normal morphology [13,22]. However, other studies indicated that DFI was not correlated with conventional fresh semen parameters $[12,23]$. In this study, no significant difference in semen parameters was found between the groups with a DFI $\leq 15 \%$ and a DFI $>15 \%$. However, there was a negative correlation between the viability rate and SDF.

Men with teratozoospermia may show significantly higher rates of ROS production, denatured DNA, and fragmented DNA [18]. Jaku-
bik-Uljasz et al. [13] reported that the DFI was positively correlated with the teratozoospermia index, the proportion of sperm with head, midpiece, and tail abnormalities, and sperm with ERC. Even in pellet swim-up samples, $14 \%$ of morphologically abnormal spermatozoa showed fragmented DNA [12]. SDF primarily occurs by defective maturation from spermatids to mature spermatozoa, abortive apoptosis within the testis, and ROS throughout the male reproductive tract $[24,25]$. Abnormal spermatozoa arise due to failed apoptosis and/or failure to repair DNA strand breaks that appear during early spermatogenesis. Spermatogonia marked for apoptosis escape this process, leading to possible anomalies that may result in abnormal sperm morphology [26]. Therefore, the relationship between sperm morphology and the SDF level is predictable. However, the details regarding the types of anomalies closely related to SDF are still unclear. Our results showed no correlation of DFI or halo type with abnormal sperm morphology in general, except for a positive correlation between small halos and the DFI in round-head sperm cells.

The occurrence of round-head sperm cells, known as globozoospermia, is a severe male infertility disorder that usually causes low fertilization and pregnancy rates $[27,28]$; furthermore, it has recently been found to show a relationship with the outcome of ART cycles (ART) $[29,30]$. Globozoospermia can result from mutations, deletion 
of gene products associated with the Golgi apparatus in cells, formation of the acroplaxome, or attachment of the acrosome to the nucleus in the sperm head [31]. Our results confirmed a relationship between round-head sperm and SDF, highlighting that round-head sperm were positively correlated with the incidence of small halos and DFI in the SCD test; further, the rate of round-head spermatozoa tended to be higher in the high-DFI group (DFI > 15\%). Dam et al. [32] observed sperm cells by electron microscopy and discovered that in partial globozoospermia, the sperm head has less condensed chromatin, part of the acrosome, and mitochondria, and that roundhead sperm are acrosome-less, with disturbed chromatin compaction. This evidence indicates that improperly compacted chromatin is related to round-head spermatozoa, suggesting that more DNA damage is present in globozoospermia [32]. Moreover, using the dUTP nick-end labeling assay and chromomycin A3 staining, another study found that the percentages of SDF and protamine-deficient spermatozoa were higher in men with globozoospermia than in fertile men [33]. Clearly, DNA fragmentation appears to be a contributor to round-head sperm cells.

Although SDF has been recognized as a supporting tool for male fertility assessment by leading research groups, studies using different assays have resulted in inconsistent conclusions [2,24]. Furthermore, the DFI cut-off values are not identical in previous studies. A DFI threshold of $15 \%$ was proposed in some recent studies that evaluated the association between the DFI and sperm morphology of infertile patients [12], embryological or clinical IVF/ICSI outcomes [34], blastocyst culture [35], or embryo development [36]. This cut-off value of $15 \%$ was also selected for treatment of SDF by micronutrient supplementation [37]. The threshold of $>15 \%$ as an abnormal DFI selected in this study should be specifically considered when drawing a conclusion. A major strength of our study is the detection of the relationship of round-head sperm with SDF and the classification of halo types. However, our conclusions were drawn from results obtained using the SCD technique alone, which constitutes a limitation of our study. Thus, further studies using different techniques should be performed to confirm this relationship.

In conclusion, the rate of sperm morphological abnormalities in semen analysis was not related to sperm DNA integrity. However, a detailed assessment of sperm morphology revealed an association with SDF with the classification of halos and the DFI using the SCD assay. Specifically, round-head sperm were found to have a strong relationship with SDF and the DFI, and a higher percentage of roundhead sperm was observed in the group of men with higher DFI. Therefore, in routine semen analysis, the detailed characteristics of abnormal sperm morphology should be described, together with a SDF assay for a better assessment of male fertility potential.

\section{Conflict of interest}

Hiep Tuyet Thi Nguyen was funded by Vingroup Joint Stock Company and was supported by the Domestic Master/ PhD Scholarship Program of Vingroup Innovation Foundation (VINIF), Vingroup Big Data Institute (VINBIGDATA), code VINIF. 2020.TS.44. No other potential conflicts of interest relevant to this article were reported.

\section{ORCID}

Hiep Tuyet Thi Nguyen ～https://orcid.org/0000-0002-8019-9608

Hong Nhan Thi Dang https://orcid.org/0000-0003-3872-5456

Thai Thanh Thi Nguyen https://orcid.org/0000-0001-9995-9948

Trung Van Nguyen

Thuan Cong Dang

Quoc Huy Vu Nguyen

Minh Tam Le

https://orcid.org/0000-0003-3363-5064

https://orcid.org/0000-0002-2657-6791

https://orcid.org/0000-0002-4744-7059

https://orcid.org/0000-0001-6225-3108

\section{Author contributions}

Conceptualization: HTTN, MTL. Data curation: HTTN, HNTD, TTTN, TVN. Formal analysis: HTTN, HNTD. Methodology: MTL, HTTN. Project administration: MTL, HTTN. Visualization: MTL, HTTN. Writing-original draft: HTTN, HNTD, TTTN, TVN, MTL. Writing-review \& editing: all authors.

\section{References}

1. Agarwal A, Mulgund A, Hamada A, Chyatte MR. A unique view on male infertility around the globe. Reprod Biol Endocrinol 2015; 13:37.

2. Agarwal A, Majzoub A, Parekh N, Henkel R. A schematic overview of the current status of male infertility practice. World J Mens Health 2020;38:308-22.

3. World Health Organization. Laboratory manual for the examination and processing of human semen. 5th ed. Geneva: World Health Organization; 2010.

4. Gunes S, Al-Sadaan M, Agarwal A. Spermatogenesis, DNA damage and DNA repair mechanisms in male infertility. Reprod Biomed Online 2015;31:309-19.

5. Marchetti F, Bishop J, Gingerich J, Wyrobek AJ. Meiotic interstrand DNA damage escapes paternal repair and causes chromosomal aberrations in the zygote by maternal misrepair. Sci Rep 2015; 5:7689.

6. Bosco L, Notari T, Ruvolo G, Roccheri MC, Martino C, Chiappetta R, et al. Sperm DNA fragmentation: an early and reliable marker of air pollution. Environ Toxicol Pharmacol 2018;58:243-9. 
7. Mostafa RM, Nasrallah YS, Hassan MM, Farrag AF, Majzoub A, Agarwal A. The effect of cigarette smoking on human seminal parameters, sperm chromatin structure and condensation. Andrologia 2018;50:e12910.

8. Aboulmaouahib S, Madkour A, Kaarouch I, Sefrioui O, Saadani B, Copin $\mathrm{H}$, et al. Impact of alcohol and cigarette smoking consumption in male fertility potential: looks at lipid peroxidation, enzymatic antioxidant activities and sperm DNA damage. Andrologia 2018;50:e12926.

9. Sharbatoghli M, Valojerdi MR, Amanlou M, Khosravi F, Jafar-abadi MA. Relationship of sperm DNA fragmentation, apoptosis and dysfunction of mitochondrial membrane potential with semen parameters and ART outcome after intracytoplasmic sperm injection. Arch Gynecol Obstet 2012;286:1315-22.

10. Karabulut A, Tekin A. Alterations in the morphology and motility of spermatozoa: relation with total sperm count. Pam Med J 2013; 6:1-4.

11. Agarwal A, Tvrda E, Sharma R. Relationship amongst teratozoospermia, seminal oxidative stress and male infertility. Reprod Biol Endocrinol 2014;12:45.

12. Ferrigno A, Ruvolo G, Capra G, Serra N, Bosco L. Correlation between the DNA fragmentation index (DFI) and sperm morphology of infertile patients. J Assist Reprod Genet 2021;38:979-86.

13. Jakubik-Uljasz J, Gill K, Rosiak-Gill A, Piasecka M. Relationship between sperm morphology and sperm DNA dispersion. Transl Androl Urol 2020;9:405-15.

14. Daris B, Goropevsek A, Hojnik N, Vlaisavljevic V. Sperm morphological abnormalities as indicators of DNA fragmentation and fertilization in ICSI. Arch Gynecol Obstet 2010;281:363-7.

15. Oehninger S, Kruger TF. Sperm morphology and its disorders in the context of infertility. F S Rev 2021;2:75-92.

16. Zhu DL, Zhang HG, Wang RX, Jiang YT, Liu RZ. Re-evaluation of the value of sperm morphology in classical in vitro fertilization in a Northeastern Chinese population. J Int Med Res 2019;47:413442.

17. Cito G, Picone R, Fucci R, Giachini C, Micelli E, Cocci A, et al. Sperm morphology: what implications on the assisted reproductive outcomes? Andrology 2020;8:1867-74.

18. Oumaima A, Tesnim A, Zohra H, Amira S, Ines Z, Sana C, et al. Investigation on the origin of sperm morphological defects: oxidative attacks, chromatin immaturity, and DNA fragmentation. Environ Sci Pollut Res Int 2018;25:13775-86.

19. Sivanarayana T, Krishna C, Prakash GJ, Krishna KM, Madan K, Rani $\mathrm{BS}$, et al. CASA derived human sperm abnormalities: correlation with chromatin packing and DNA fragmentation. J Assist Reprod Genet 2012;29:1327-34.

20. Samplaski MK, Dimitromanolakis A, Lo KC, Grober ED, Mullen B,
Garbens A, et al. The relationship between sperm viability and DNA fragmentation rates. Reprod Biol Endocrinol 2015;13:42.

21. Belloc S, Benkhalifa M, Cohen-Bacrie M, Dalleac A, Chahine H, Amar $\mathrm{E}$, et al. Which isolated sperm abnormality is most related to sperm DNA damage in men presenting for infertility evaluation. $J$ Assist Reprod Genet 2014;31:527-32.

22. Sivanarayana T, Ravi Krishna C, Jaya Prakash G, Krishna KM, Madan K, Sudhakar G, et al. Sperm DNA fragmentation assay by sperm chromatin dispersion (SCD): correlation between DNA fragmentation and outcome of intracytoplasmic sperm injection. Reprod Med Biol 2013;13:87-94.

23. Xie D, Lu C, Zhu Y, Zhu S, Yang EJ, Jin X. Analysis on the association between sperm DNA fragmentation index and conventional semen parameters, blood microelements and seminal plasma ROS in male patients with infertility. Exp Ther Med 2018;15:5173-6.

24. Agarwal A, Majzoub A, Baskaran S, Panner Selvam MK, Cho CL, Henkel $R$, et al. Sperm DNA fragmentation: a new guideline for clinicians. World J Mens Health 2020;38:412-71.

25. Ribas-Maynou J, Yeste M. Oxidative stress in male infertility: causes, effects in assisted reproductive techniques, and protective support of antioxidants. Biology (Basel) 2020;9:77.

26. Sakkas D, Seli E, Bizzaro D, Tarozzi N, Manicardi GC. Abnormal spermatozoa in the ejaculate: abortive apoptosis and faulty nuclear remodelling during spermatogenesis. Reprod Biomed Online 2003;7:428-32.

27. Kullander S, Rausing A. On round-headed human spermatozoa. Int J Fertil 1975;20:33-40.

28. Dam AH, Feenstra I, Westphal JR, Ramos L, van Golde RJ, Kremer JA. Globozoospermia revisited. Hum Reprod Update 2007;13:6375.

29. Kochhar PK, Ghosh P. Intracytoplasmic sperm injection with assisted oocyte activation resulting in successful pregnancies and live birth in couples with globozoospermia: a report of two cases. $J$ Hum Reprod Sci 2018;11:72-4.

30. Zhang ZQ, Long SG, Huang ZH, Xin CL, Wu QF. Different outcomes after intracytoplasmic sperm injection without oocyte activation in two patients with different types of globozoospermia. Andrologia 2016;48:116-20.

31. Modarres P, Tavalaee M, Ghaedi K, Nasr-Esfahani MH. An overview of the globozoospermia as a multigenic identified syndrome. Int J Fertil Steril 2019;12:273-7.

32. Dam AH, Ramos L, Dijkman HB, Woestenenk R, Robben $H$, van den Hoven L, et al. Morphology of partial globozoospermia. J Androl 2011;32:199-206.

33. Eskandari N, Tavalaee M, Zohrabi D, Nasr-Esfahani MH. Association between total globozoospermia and sperm chromatin defects. Andrologia 2018;350:e12843. 
34. Green KA, Patounakis G, Dougherty MP, Werner MD, Scott RT Jr, Franasiak JM. Sperm DNA fragmentation on the day of fertilization is not associated with embryologic or clinical outcomes after IVF/ICSI. J Assist Reprod Genet 2020;37:71-6.

35. Broussard AL, Leader B, Tirado EE, Russell H, Beydoun HA, Colver R, et al. High sperm DNA fragmentation index (DFI) on the day of oocyte retrieval is correlated with lower fertilization and slower development to the blastocyst stage for trophectoderm biopsy. Fertil Steril 2018;110:E295.
36. Alvarez Sedo C, Bilinski M, Lorenzi D, Uriondo H, Noblia F, Longobucco $V$, et al. Effect of sperm DNA fragmentation on embryo development: clinical and biological aspects. JBRA Assist Reprod 2017;21:343-50.

37. Eidenberger F, Huber KL, Fuchs P, Simkovicova N, Imhof M. Measured with sperm chromatin dispersion (SCD) technique: will DNA integrity in spermatozoa increase after micronutrient supplementation? Comparative controlled study. Fertil Reprod 2019; 1:136-40. 